$06,10,11$

\title{
Фазовые переходы в гафнате свинца в условиях высокого давления
}

\author{
(C) М.А. Князева ${ }^{1}$, Д.А. Андроникова ${ }^{1,2}$, Г.А. Литягин ${ }^{1}$, Ю.А. Бронвальд ${ }^{1,2}$, P. Parisiades ${ }^{3}$, \\ A. Majchrowski ${ }^{4}$, K. Roleder ${ }^{5}$, А.В. Филимонов ${ }^{1}$, Р.Г. Бурковский ${ }^{1}$ \\ ${ }^{1}$ Санкт-Петербургский политехнический университет Петра Великого, \\ Санкт-Петербург, Россия \\ ${ }^{2}$ Физико-технический институт им. А.Ф. Иофффе РАН, \\ Санкт-Петербург, Россия \\ ${ }^{3}$ European Synchrotron Radiation Facility (ESRF), \\ Grenoble, France \\ ${ }^{4}$ Institute of Applied Physics, Military University of Technology, \\ Warszawa, Poland \\ ${ }^{5}$ Institute of Physics, University of Silesia, \\ Chorzów, Poland \\ E-mail: kniazeva.maria225@yandex.ru
}

Поступила в Редакцию 14 марта 2019 г.

В окончательной редакции 24 апреля 2019 г.

Принята к публикации 14 мая 2019 г.

\begin{abstract}
Исследовано влияние гидростатического давления на фазовые переходы в гафнате свинца $\left(\mathrm{PbHfO}_{3}\right)$ методами дифракции и диффузного рассеяния рентгеновского излучения. Измерения проведены в интервале температур $182<T<316^{\circ} \mathrm{C}$ и в интервале давлений $1.6<P<2.6 \mathrm{GPa}$. Выявлены четыре области, характеризуемые различными дифракционными картинами и соответствующие антисегнетоэлектрической фазе, фазам с длинноволновой модуляцией свинцовой подрешетки и фазам с различными искажениями кислородной подрешетки. В температурной области, находящейся непосредственно над областью фаз с длинноволновой модуляцией, выявлен температурно-зависимый максимум в распределении диффузного рассеяния, указывающий на то, что образование таких модуляцией обусловлено конденсацией несоразмерной мягкой моды.
\end{abstract}

Ключевые слова: антисегнетоэлектрики, фазовые переходы, дифракция синхротронного излучения, гафнат свинца, гидростатическое давление.

DOI: 10.21883/FTT.2019.10.48252.421

\section{1. Введение}

Антисегнетоэлектрические (АСЭ) материалы начали привлекать внимание исследователей в 50-х годах XX века, с момента открытия первого АСЭ кристалла цирконата свинца, $\mathrm{PbZrO}_{3}$ [1]. Антисегнетоэлектрики нашли широкое применение в промышленности: их твердые растворы с сегнетоэлектриками используются в устройствах различного назначения, включая пьезоэлектрические и пироэлектрические преобразователи, электрооптические устройства [2-4]. Интерес к антисегнетоэлектрикам растет в связи с новыми способами их практического применения в энергозапасающих и запоминающих устройствах [5-7].

Механизмы фазовых переходов в свинец-содержащих антисегнетоэлектриках остаются на сегодняшний день недостаточно изученными. Основные трудности связаны с наличием нескольких параметров порядка, которые соответствуют разным неприводимым представлениям. В частности в в модельном антисегнетоэлектрике $\mathrm{PbZrO}_{3}$ основными типами искажений $\mathrm{AC} Э$ фазы являются упорядоченные, противоположно направленные смещения ионов свинца и антифазные повороты октаэдрических кислородных групп [8]. Взаимо- действие между этими параметрами порядка трактуется на сегодняшний день неоднозначно: существуют указания как на положительную [9], так и на отрицательную [10] биквадратичную связь между ними. Также обсуждается влияние взаимодействия этих параметров порядка с третьей степенью свободы, известной как мода S4, которая способствует реализации АСЭ структуры вместо альтернативных структур с близкой энергией [11]. Проблема термодинамического описания АСЭ кристаллов в терминах взаимодействующих параметров порядка является в настоящее время нерешенной и требует дополнительных экспериментальных исследований для выработки самосогласованной модели.

В этой связи представляет интерес определение фазовых диаграмм антисегнетоэлектриков в пространстве температура-давление. Недавно было показано, что при увеличении давления последовательность фазовых переходов в антисегнетоэлектрике $\mathrm{PbZrO}_{3}$ радикально меняется. Вместо одиночного перехода из кубической в АСЭ фазу наблюдается серия из трех переходов, среди которых есть несоразмерный фазовый переход [12].

Родственный кристалл, $\mathrm{PbHfO}_{3}$, обладает еще более сложной фазовой диаграммой [13], поэтому его иссле- 
дование представляет очевидный научный интерес. Для этого соединения были идентифицированы два фазовых перехода при температурах $T=163^{\circ} \mathrm{C}$ и $T=215^{\circ} \mathrm{C}[14]$. Первый переход разделяет низкотемпературную и промежуточную АСЭ фазы (обозначаются в литературе как А1 и А2), второй переход - промежуточную АСЭ фазу и высокотемпературную параэлектрическую (кубическую) фазу. Низкотемпературная $\mathrm{AC} Э$ фаза в $\mathrm{PbHfO}_{3}$, при атмосферном давлении, изоструктурна [15-17] низкотемпературной АCЭ фазе в $\mathrm{PbZrO}_{3}$ [18]. Недавно установлено, что промежуточная АСЭ фаза, при атмосферном давлении, характеризуется длинноволновой модуляцией в свинцовой подрешетке, волновой вектор модуляции $\mathbf{q}=(0.15,0.15,0)$ в псевдокубических координатах, а также антифазными поворотами кислородных октаэдров [19].

При приложении электрического поля [20] и внешнего давления [21,22] последовательность фазовых переходов в $\mathrm{PbHfO}_{3}$ модифицируется. В работах [21,22] методами дифракции синхротронного излучения и рамановской спектроскопии исследованы фазовые переходы, индуцированные давлением при комнатной температуре и обнаружен новый фазовый переход в области весьма высоких давлений $~ 45 \mathrm{GPa}$. В работе [13] получена фазовая диаграмма температура-давление гафната свинца на основании диэлектрических измерений. Установлено, что помимо характерных для атмосферного давления фаз наблюдается еще одна фаза, расположенная между промежуточной АСЭ и параэлектрической фазами, известная как $\beta$-фаза [13].

В нашей работе [23], выполненной методом дифракции синхротронного излучения, выполнен анализ сверхструктурных отражений в $\mathrm{PbHfO}_{3}$ в области высоких давлений и температур. Обнаружено, что при высоких давлениях антифазные повороты кислородных октаэдров возникают при более высоких температурах, чем искажения в свинцовой подсистеме, в отличие от ситуации при атмосферном давлении, где эти два типа искажений возникают одновременно. Зарегистрированы сверхструктурные отражения, нехарактерные для свинецсодержащих антисегнетоэлектриков, природа которых на данный момент не установлена. Также на сегодняшний день остаются неясными механизмы обнаруженных под давлением фазовых переходов.

В данной работе исследована температурная зависимость обобщенной статической восприимчивости в промежуточных фазах $\mathrm{PbHfO}_{3}$ при помощи рентгеновского диффузного рассеяния (ДР). Показано, что при охлаждении кристалла под высоким давлением образованию структур с длинноволновой модуляцией в свинцовой подсистеме предшествует рост обобщенной восприимчивости на ненулевом волновом векторе, что позволяет предположить определяющую роль несоразмерной мягкой моды в формировании таких структур.

Дальнейшее изложение организовано следующим образом. В разделе „Методы“ описаны методы исследования - дифракция и ДР синхротронного излучения с применением алмазных наковален. В разделе „Результаты“ изложены экспериментальные наблюдения, проведена классификация областей в пространстве температурадавление по наблюдаемым сверхструктурным отражениям и характерным особенностям ДР. В разделе „Обсуждение“ предложена интерпретация экспериментальных наблюдений в контексте их связи со структурными фазовыми переходами.

\section{2. Методы}

Мы использовали в качестве образцов монокристаллы $\mathrm{PbHfO}_{3}$, выращенные методом, описанным в работе [24]. Для проведения измерений был приготовлен кристалл размерами $30 \times 30 \times 30 \mu \mathrm{m}$ путем шлифовки и последующего травления в соляной кислоте. Для работы с давлением применялась ячейка с алмазными наковальнями (DAC) по схеме, аналогичной той, которая использовалась в работе [12]. В качестве среды, передающей давление, был использован неон, который, являясь одним из наиболее легких благородных газов, приводит к сравнительно малому паразитному фону. Давление определялось стандартным методом измерения флуоресцентного сигнала от кристалла рубина, помещенного в ячейку вместе с образцом. Температура образца оценивалась по температуре алмаза, непосредственно измеряемой при помощи термопары.

Рентгеновские измерения проведены на Европейском синхротронном источнике (ESRF), на экспериментальной станции ID27. Картины рассеяния регистрировались при непрерывном вращении образца с шагом в $1^{\circ}$ и экспозицией $1 \mathrm{~s}$. Измерения выполнялись в температурном диапазоне от 182 до $330^{\circ} \mathrm{C}$ и диапазоне давлений от 1.6 до $2.6 \mathrm{GPa}$. Регистрация рассеянного излучения выполнялась с помощью позиционно чувствительного детектора Perkin Elmer.

Измерения проведены в цикле нагрева. Экспериментальные точки расположены на линии $P=1.6+$ $+0.0075 \mathrm{GPa} /{ }^{\circ} \mathrm{C}\left(T-185^{\circ} \mathrm{C}\right)$, что связано со спонтанным изменением давления при контролируемом изменении температуры.

Восстановление распределений интенсивности в обратном пространстве осуществлено с использованием подхода [25]. Анализ искажений структуры проводился по признаку наличия или отсутствия сверхструктурных отражений, соответствующих определенным точкам зоны Бриллюэна. Далее мы используем стандартные обозначения таких точек: $R(1 / 2 \pm h, 1 / 2 \pm k, 1 / 2 \pm l), \quad M(1 / 2 \pm h, k, 1 / 2 \pm l)$, $X(1 / 2 \pm h, k, l)$ и $\Sigma(1 / 4 \pm h, k, 1 / 4 \pm l)$, где $h, k, l-$ индексы Миллера. Для обозначения сверхструктурных отражений, соответствующих длинноволновым модуляциям различного периода, мы используем обозначения $\Sigma_{i}\left(\xi_{i} \pm h, k, \xi_{i} \pm l\right)$, где $i=1,2$, а $\xi_{i}$ - вещественные числа. 


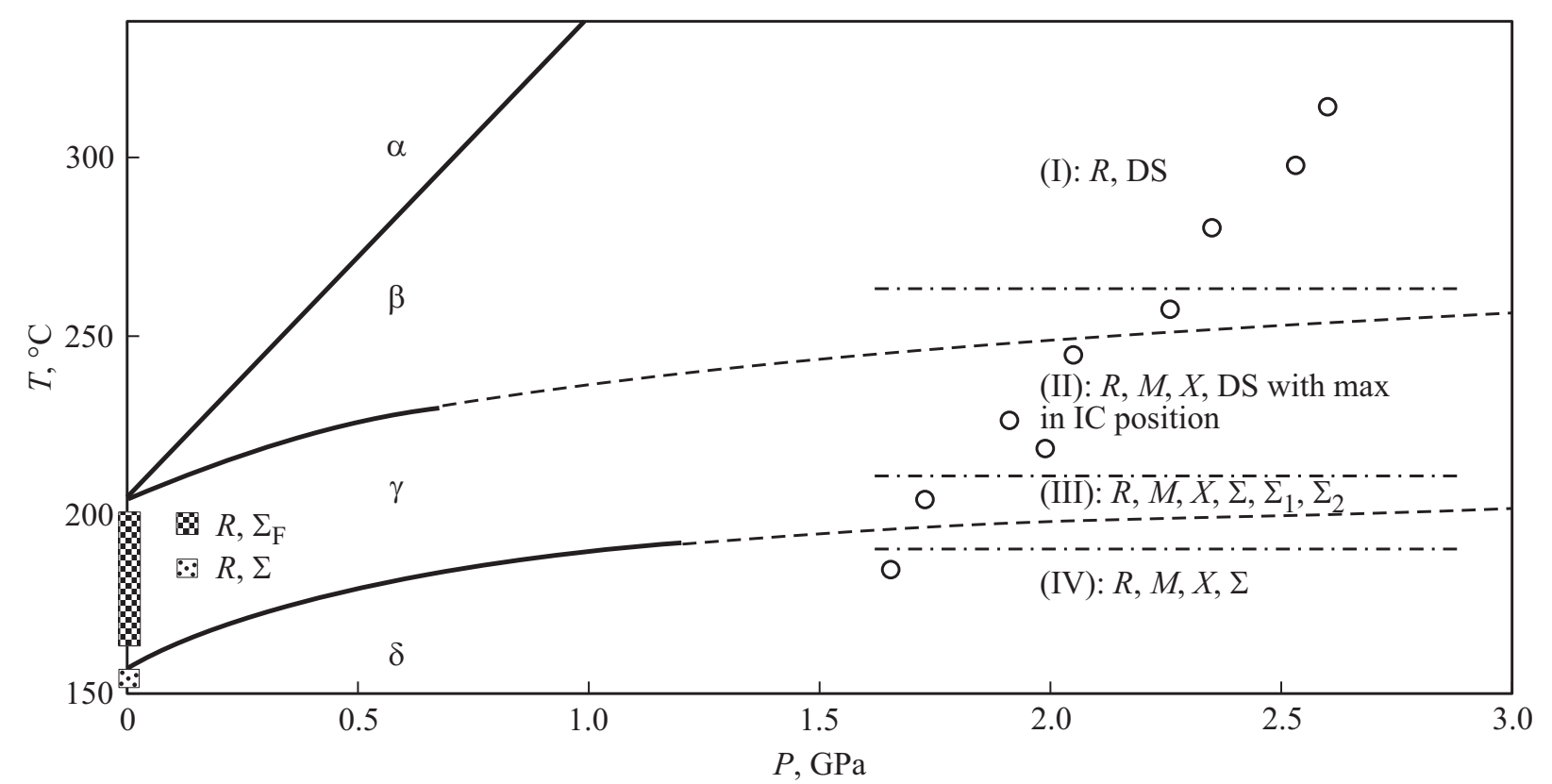

Рис. 1. Фазовая диаграмма гафната свинца в пространстве давление-температура. Круги - экспериментальные точки, в которых были проведены измерения; сплошные линии — фазовые границы, полученные в работе [13]; пунктирные линии их экстраполяция в релевантную область давлений. Обозначения фаз $\alpha, \beta, \gamma, \delta$ взяты из работы [13]. Штрихпунктирные линии разграничивают области I-IV, характеризуемые различными дифракционными картинами. Дифракционные особенности данных областей (типы наблюдаемых сверхструктурных отражений, особенности ДР) перечислены на рисунке. Маркером $\Sigma_{F}$ обозначены длинноволновые модуляции с волновым вектором $\mathbf{q}_{\mathbf{F}}=(0.15,0.15,0)$ из работы [19].

Поведение обобщенной восприимчивости по отношению к длинноволновым модуляциям было изучено методом диффузного рассеяния (ДР). Поскольку соответствующий сигнал проинтегрирован по энергии, интенсивность ДР пропорциональна Фурье-образу коррелятора мгновенных смещений, который, в свою очередь, пропорционален произведению соответствующей компоненты статической обобщенной восприимчивости и температуры: $I^{\text {Diff }} \sim k T \chi^{\text {Stat }}[26]$. В случае свинецсодержащих антисегнетоэлектриков основной компонентой ДР вблизи линии $(\xi, \xi, 0)$ является рассеяние на флуктуациях параметра порядка, связанного с поперечными волнами смещений ионов свинца $[10,12]$. Таким образом, посредством измерения температурных зависимостей диффузного рассеяния в этой области зоны Бриллюэна можно оценить температурную зависимость обобщенной статической восприимчивости по отношению к длинноволновым модуляциям, что было использовано для выявления несоразмерной мягкой моды в одной из высокотемпературных фаз.

\section{3. Результаты}

Точки на диаграмме температура-давление, для которых проведены измерения, указаны на рис. 1 совместно с фазовыми границами, определенными в области малых давлений при помощи диэлектрических измерений [13].
В рассматриваемом диапазоне идентифицировано четыре области (I-IV), с качественно различающимися дифракционными картинами, разделенные на рис. 1 штрих-пунктирными линиями. Для каждой области приведен список характерных сверхструктурных отражений. Имеющихся дифракционных данных недостаточно для определения наклонов границ областей, поэтому мы принимаем их условно горизонтальными в соответствии с малым наклоном экстраполированных фазовых границ работы [13]. Мы описываем результаты при понижении температуры, поскольку при высоких температурах наблюдаются более высокосимметричные фазы и, соответственно, более простые картины дифракции.

Область I $\left(T \gtrsim 270^{\circ} \mathrm{C}\right)$ характеризуется наличием сверхструктурных отражений, соответствующих только $R$-точкам. ДР в этой области хорошо различимо и существенно превосходит уровень фона (рис. $2(a, b)$ ). Интенсивность ДР монотонно увеличивается при приближении к центру зоны Бриллюэна (рис. 2, $(a, b)$ ). Распределение интенсивности не содержит локальных максимумов или иных особенностей на конечных волновых векторах.

Область II $\left(\sim 210<T \lesssim 275^{\circ} \mathrm{C}\right)$ характеризуется отражениями, которые соответствуют $R$-, $M$ - и $X$-точкам. При этом наблюдается систематическое погасание рефлексов, соответствующих $M$-точкам с координатами $(H, K, L)$, где $K$ - целое, а $H$ и $L-$ полуцелые, связанные соотношением $H= \pm L$. Помимо появления новых сверхструктурных отражений, изменяется харак- 

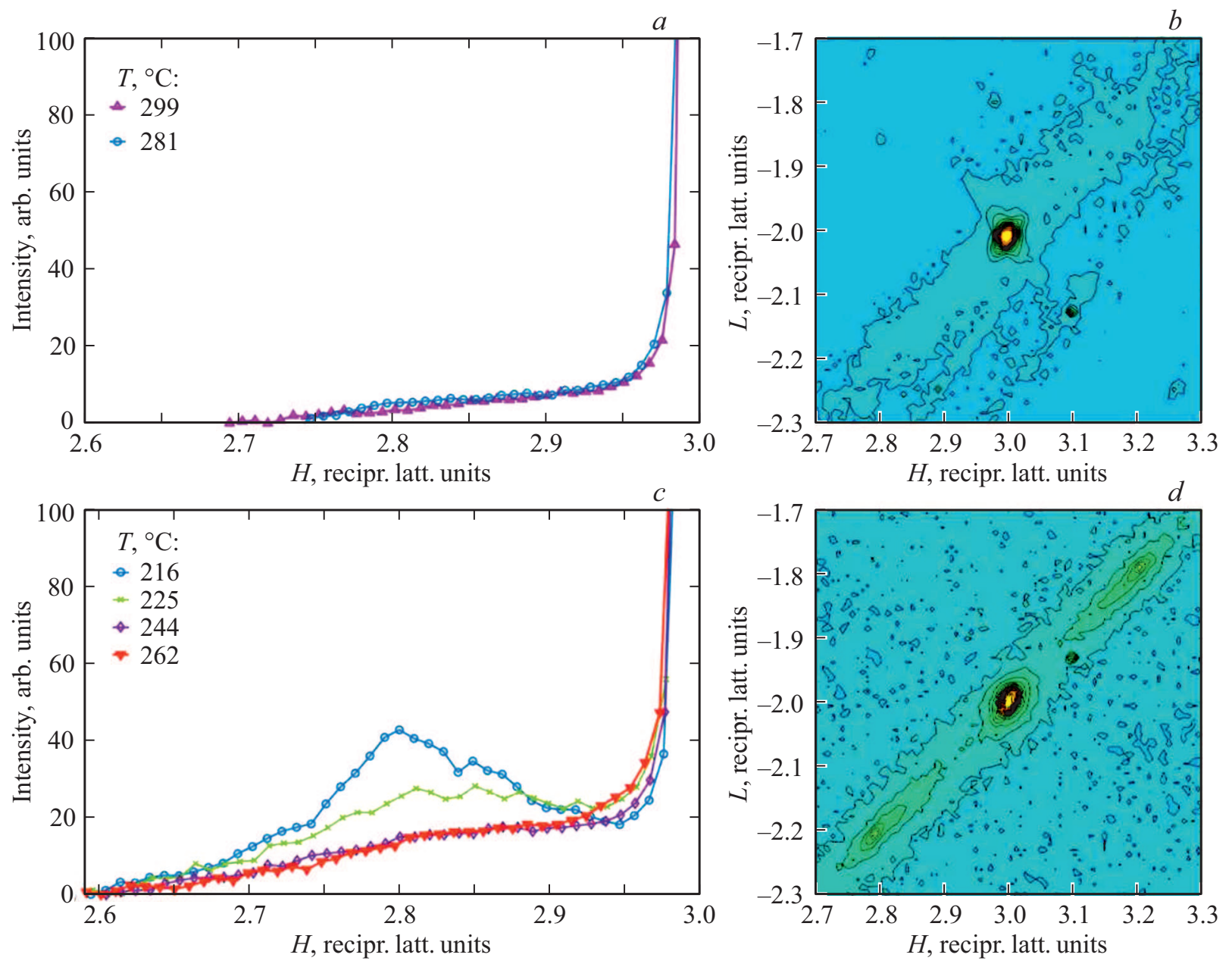

Рис. 2. Профили интенсивности ДР в окрестности узла $(30-2)$ в направлении [101] и его двумерные распределения в плоскости $H 0 L$ для областей I $(a, b)$ и II $(c, d)$.

тер распределения ДР в данной области: для ряда температур распределение содержит температурно-зависимый максимум на ненулевом приведенном волновом векторе, $\mathbf{q}_{\max }($ рис. $2,(c, d))$. Данный максимум хорошо различим на распределениях при $T=225^{\circ} \mathrm{C}\left(\mathbf{q}_{\max } \sim(0.2,0.2,0)\right)$ и $T=216^{\circ} \mathrm{C}$. При удалении от максимума интенсивность ДР спадает значительно медленнее вдоль направления, параллельного вектору $\mathbf{q}_{\max }$, чем вдоль направления, перпендикулярного $\mathbf{q}_{\mathbf{m a x}}$ (рис. 2,d). При более высоких температурах в этой же области максимума не наблюдается, но виден слабый округлый излом в той же области зоны Бриллюэна. С уменьшением температуры интенсивность ДР возрастает.

Область III введена нами на основании анализа результатов, полученных при $T=202^{\circ} \mathrm{C}$, где наблюдается дифракционная картина, которая отличается от картины в других областях (рис. $3,(a, b))$. К уже имеющимся в области II сверхструктурным отражениям $R$-, $M$ - и $X$-типа добавляются отражения $\Sigma$-, $\Sigma_{1}$ - и $\Sigma_{2}$-типа с координатами $\left(\xi_{i} \pm h, 0, \xi_{i} \pm l\right)$, где $\xi_{i}$ принимает значения $\xi \sim 0.25, \xi_{1} \sim 0.22$ и $\xi_{2} \sim 0.17$. Также при $q \sim 0.34$ можно увидеть рефлексы второго порядка, соответствующие вектору модуляции $\xi_{2} \sim 0.17$. Ширину данной области по температуре, на основании имеющихся данных, можно оценить как не превосходящую $20^{\circ} \mathrm{C}$.

Область IV $\left(T \leq 182^{\circ} \mathrm{C}\right)$ характеризуется наличием сверхструктурных отражений $R$-, $M$-, $X$ - и $\Sigma$-типа (рис. $3,(c, d)$ ). Выделить сильную компоненту ДР в направлениях [llll] в д данной области, в отличие от ранее рассмотренных областей, не удается в силу малости интенсивности ДР по сравнению с уровнем фона.

\section{4. Обсуждение}

Согласно работе [13], ожидается присутствие трех различных фаз в исследованной нами области давлений и температур (см. рис. 1): $\delta$ - низкотемпературная антисегнетоэлектрическая фаза; $\gamma$ - фаза с длинноволновой модуляцией и анти-фазными поворотами кислородных октаэдрических групп (согласно данным [19], см. рис. 1) и $\beta$-фаза. Наши данные по дифракции и ДР указывают на большее количество различных фаз в изученном интервале температур и давлений. 

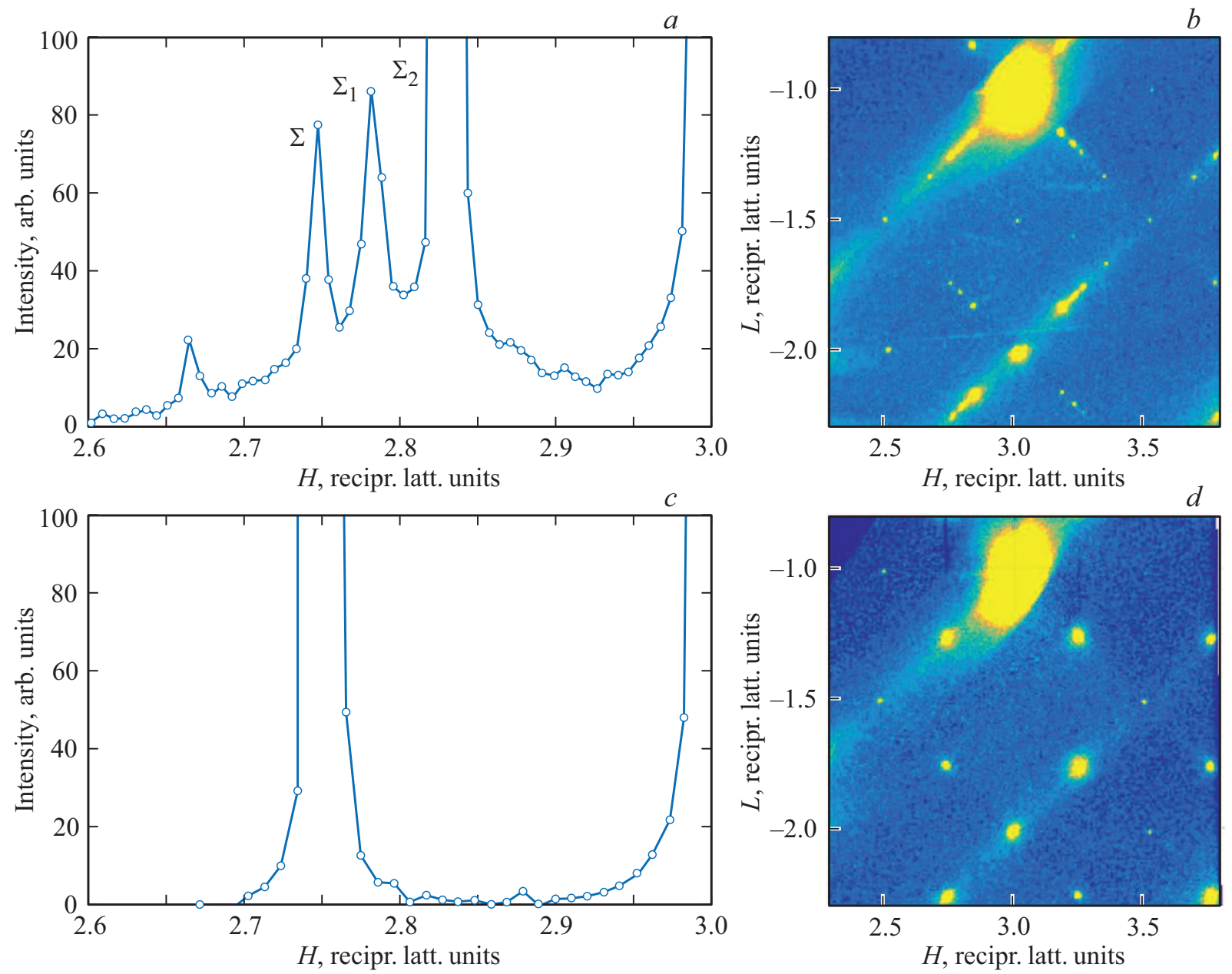

Рис. 3. Профили интенсивности рассеяния в окрестности узла $(30-2)$ в направлении [1 01 1] и его двумерные распределения в плоскости $H 0 L$ для областей III $(a, b)$ и $\mathrm{IV}(c, d)$.

Границу между областями III и IV можно соотнести с фазовым переходом из фазы $\delta$ в фазу $\gamma$, поскольку в случае данной границы не наблюдается существенных расхождений с экстраполяцией фазовых границ из диэлектрических исследований. Таким образом, область IV соответствует $\delta$-фазе (низкотемпературная АСЭ фаза) в работе [13]. Данные нашего исследования подтверждают наличие обоих типов ионных смещений, характерных для АСЭ фазы $[18,27]-$ антипараллельных смещений ионов свинца, приводящих к отражениям $\Sigma$ - и $M$-типа, и антифазных поворотов кислородных октаэдров, приводящих к отражениям $R$-типа.

В то же время, наблюдаются расхождения, которые выражаются в обнаружении нетипичных для этой фазы отражений $X$-типа, природа которых на данный момент не ясна. Аналогичные наблюдения в родственных материалах неизвестны. Можно предположить, что отражения $\mathrm{X}$-типа появляются в результате дополнительной анти-фазной компоненты в смещениях ионов свинца. Аналогичная компонента смещений ионов кальция характерна для $\mathrm{CaTiO}_{3}$ [28]. Неясным остается причина появления этих искажений. С одной стороны, эти искажения, отсутствующие в $\delta$-фазе при атмосферном давлении [15], могут появляться в результате дополнительного фазового перехода в промежутке между атмосферным давлением и $P=1.65 \mathrm{GPa}$. Это подразумевает появление дополнительной фазовой границы. С другой стороны, нельзя исключить возможности, что эти дополнительные искажения возникают как результат дефектов в образце, предположительно связанных с попаданием атомов благородного газа (среды, передающей давление) в междоузлия кристалла под действием высоких давлений и температур. Уточнение причины дополнительных рефлексов требует дополнительных экспериментов.

Области II и III схожи в поведении отражений $M$-типа: в этих областях, в отличие от области IV, наблюдается систематическое погасание отражений $M$-типа с координатами $(H, K, L)$, где $K-$ целое, а $H$ и $L-$ полуцелые, связанные соотношением $H= \pm L$. Именно такой закон погасания характерен для случая, когда появление сверхструктурных отражений $M$-типа вызвано поворотами кислородных октаэдров [28]. Таким образом, отражения, наблюдаемые нами в области IV и в областях II и III, имеют различную природу. В первом 
случае это в основном отражения второго порядка, соответствующие антисегнетоэлектрической модуляции, во втором случае - антиферродисторсионные искажения, связанные с кислородной подсистемой.

В области III мы зарегистрировали отражения с координатами вида $(\xi, \xi, 0)$, где $\xi \sim 0.17, \sim 0.22$ и 0.25 . По аналогии с известной структурой фаз А1 и А2 при атмосферном давлении, данные отражения соответствуют поперечным волнам смещений, в основном - ионов свинца, с периодами $1 / \xi \sim 5.88,4.55$ и 4, соответственно. Наибольший из данных периодов $(\sim 5.88)$ близок, но отличается от такового для $\gamma$-фазы при атмосферном давлении $(\sim 6.66)$ [19]. Указанные выше три типа отражений зарегистрированы в нашем эксперименте одновременно. Такое состояние, при котором одновременно реализуются три различных периода модуляции свинцовой подсистемы, может быть вызвано тем, что в кристалле одновременно присутствуют домены трех разных кристаллических фаз. В принципе, это возможно вследствие неравномерного распределения давления или температуры в кристалле, однако такой сценарий представляется нам маловероятным в силу высокой гидростатичности неона, а также в силу малости образца, подразумевающей высокую однородность по температуре. Альтернативным сценарием, представляющимся более вероятным, видится сосуществование трех фаз в момент проведения измерений в силу медленной кинетики фазовых переходов, при которой домены последующих фаз при нагреве еще не полностью вытеснили домены предыдущих фаз. Формально нельзя исключить возможность тройной точки при температуре $202^{\circ} \mathrm{C}$ и давлении $1.75 \mathrm{GPa}$, хотя вероятность этого весьма невелика.

Применение метода ДР позволило выявить наиболее вероятный механизм формирования фаз с длинноволновой модуляцией в области III. В области II, расположенной над областью III по температуре, наблюдается максимум интенсивности диффузного рассеяния на ненулевом волновом векторе. Данный максимум соответствует максимуму обобщенной восприимчивости [26] и, как следствие, минимуму жесткости для образования волн смещений, описываемых таким волновым вектором. При понижении температуры интенсивность диффузного рассеяния возрастает, что связано с уменьшением несоразмерной жесткости. Такой характер зависимости интенсивности от температуры позволяет предположить, что между областями II и III существует фазовый переход, близкий к переходу второго рода, для которого роль параметра порядка играют несоразмерные модуляции решетки. Температурно-зависимый максимум диффузного рассеяния следует интерпретировать как наличие несоразмерной мягкой моды, аналогично ситуации в $\mathrm{PbZrO}_{3}$ под давлением [12].

Полученные дифракционные результаты указывают на то, что наиболее высокотемпературная из исследованных фаз является антиферродисторсионной. При этом данная фаза появляется только при высоких давлениях (при атмосферном давлении в этом температурном интервале - кубическая фаза [13]). Таким образом, увеличение давления дестабилизирует кубическую фазу по отношению к антифазным поворотам кислородных октаэдров (мода $\Gamma_{25}$ в обозначениях [29]). Полагая, что соответствующий фазовый переход близок к переходу второго рода, можно утверждать, что увеличение давления приводит к уменьшению антиферродисторсионной жесткости, входящей в разложение Ландау в виде $\alpha_{\mathrm{AFD}}(P, T) \eta^{2}$, где $\eta-$ амплитуда поворотов. Это находится в согласии с недавними результатами первопринципного анализа, указывающими на склонность к такому поведению в кристаллах со сравнительно малыми факторами толерантности [30], к каковым относится $\mathrm{PbHfO}_{3}[31]$.

Несоразмерная мягкая мода в области III является необычной в следующем аспекте. Как правило, в классических несоразмерных кристаллах мягкая мода наблюдается в высокотемпературной высокосимметричной фазе, свободной от наличия дополнительных параметров порядка, сильно изменяющихся с температурой [32]. $\mathrm{B} \mathrm{PbHfO}_{3}$ ситуация отличается в силу наличия таких параметров порядка (искажения кислородной подсистемы). Уменьшение несоразмерной жесткости при охлаждении может быть вызвано не только „естественными причинами“ смягчения мод [33], но и увеличением амплитуды других параметров порядка ( $\eta$ и, возможно, другие параметры порядка, связанные с отражениями в $M$ - и $X$-точках) при наличии отрицательной биквадратичной связи в форме $\delta \eta^{2}|A|^{2}$, где $|A|$ - амплитуда несоразмерной модуляции [10]. Масштаб такого влияния предстоит выяснить в дальнейших исследованиях.

\section{5. Заключение}

Картина переходов в $\mathrm{PbHfO}_{3}$ под давлением существенно сложнее, чем при атмосферном давлении. Оказываются разделенными по температуре моменты образований искажений, связанных с модами $\Gamma_{25}$ и $\Sigma_{3}$ в отличие от ситуации при атмосферном давлении, где данные искажения возникают одновременно. В промежуточной фазе, возникающей только под давлением, обнаружена несоразмерная мягкая мода, конденсация которой приводит к формированию длиннопериодических структур. Полученные результаты представляют собой хороший экспериментальный базис для развития теории свинец-содержащих антисегнетоэлектриков.

\section{Финансирование работы}

Работа выполнена при поддержке Российского научного фонда (№ 17-72-20083).

\section{Конфликт интересов}

Авторы заявляют об отсутствии конфликта интересов. 


\section{Список литературы}

[1] E. Sawaguchi, H. Maniwa, S. Hoshino. Phys. Rev. 83, 5, 1078 (1951).

[2] G.H. Haertling. J. Am. Ceram. Soc. 82, 4, 797 (1999).

[3] J.F. Scott. Science 315 5814, 954 (2007).

[4] C. Heremans, H.L. Tuller. J. Eur. Ceram. Soc. 19, 6-7, 1133 (1999).

[5] M.H. Park, C.S. Hwang. Ferroelectric-Gate Field Effect Transistor Memories, Springer, Dordrecht (2016).

[6] B. Xu, J. Íñiguez, L. Bellaiche. Nature Commun. 8, 15682 (2017).

[7] Z. Liu, T. Lu, J. Ye, G. Wang, X. Dong, R. Withers, Y. Liu. Adv. Mater. Technol. 3, 9, 1800111. (2018)

[8] R.W. Whatmore, A.M. Glazer. J. Phys. C 12, 8, 1505 (1979).

[9] J. Íñiguez, M. Stengel, S. Prosandeev, L. Bellaiche. Phys. Rev. B, 90, 22, 220103 (2014).

[10] A.K. Tagantsev, K. Vaideeswaran, S.B. Vakhrushev, A.V. Filimonov, R.G. Burkovsky, A. Shaganov, D. Andronikova, A.I. Rudskoy, A.Q.R. Baron, H. Uchiyama, D. Chernyshov, A. Bosak, Z. Ujma, K. Roleder, A. Majchrowski, J.-H. Ko, N. Setter. Nature Commun. 4, 2229 (2013).

[11] J. Hlinka, T. Ostapchuk, E. Buixaderas, C. Kadlec, P. Kuzel, I. Gregora, J. Kroupa, M. Savinov, A. Klic, J. Drahokoupil. Phys. Rev. Lett. 112, 19, 197601 (2014).

[12] R.G. Burkovsky, I. Bronwald, D. Andronikova, B. Wehinger, M. Krisch, J. Jacobs, D. Gambetti, K. Roleder, A. Majchrowski, A.V. Filimonov, A.I. Rudskoy, S.B. Vakhrushev, A.K. Tagantsev. Sci. Rep. 7, 41512 (2017).

[13] G.A. Samara. Phys. Rev. B 1, 3777 (1970).

[14] G. Shirane, R. Pepinsky. Phys. Rev. 91, 4, 812 (1953).

[15] D.L. Corker, A.M. Glazer, W. Kaminsky, R.W. Whatmore, J. Dec, K. Roleder. Acta Cryst. B 54, 1, 18 (1998).

[16] M.F. Kuprianov, S.M. Zaitsev, E.S. Gagarina, E.G. Fesenko. Phase Trans. 4, 1, 55 (1983).

[17] H. Fujishita, A. Ogawaguchi, S. Katano. J. Phys. Soc. Jpn. 77, 6, 064601 (2008).

[18] V. Madigout, J.L. Baudour, F. Bouree, C. Favotto, M. Roubin, G. Nihoul. Phil. Mag. A 79, 4, 847 (1999).

[19] H. Fujishita, K. Kato, E. Nishibori, M. Takata, M. Sakata, S. Katano . J. Phys. Soc. Jpn 87, 12, 124603 (2018).

[20] O.E. Fesenko, L.E. Balyunis. Ferroelectrics 29, 1, 95 (1980).

[21] L.C. Ming, A. Jayaraman, S.R. Shieh, Y.H. Kim, M.H. Manghnani. J. Phys. Chem. Sol. 55, 11, 1213 (1994).

[22] A. Jayaraman, S.K. Sharma, L.C. Ming, S.Y. Wang. J. Phys. Chem. Sol. 55, 11, 1207 (1994).

[23] M. Knjazeva, Y. Bronwald, D. Andronikova, G. Lityagin, A. Bosak, P. Paraskevas, , K. Roleder, A. Majchrowski, A. Fotiadi, A. Filimonov, R. Burkovsky. Defect Diffus. Forum 386, 149 (2018).

[24] R.G. Burkovsky, D. Andronikova, Y. Bronwald, M. Krisch, K. Roleder, A. Majchrowski, A.V. Filimonov, A.I. Rudskoy, S.B. Vakhrushev. J. Phys.: Condens. Matter 27, 33, 335901 (2015).

[25] M.A. Knyazeva, Yu.A. Bronvald, A.A. Bosak, R.G. Burkovsky, D.A. Andronikova, G.A. Lityagin, S.B. Vakhrushev, G.A. Politova, A.V. Filimonov. IX Int. Sci. Tech. Conf. „Micro- and nanotechnology in electronics“. Nalchik (2017).

[26] А. Брус, Р. Каули. Структурные фазовые переходы Мир, M. (1984). $408 \mathrm{c}$.
[27] R.W. Whatmore, A.M. Glazer. J. Phys. C 12, 8, 1505 (1979).

[28] A.M. Glazer. Acta Cryst. A 31, 6, 756 (1975).

[29] R.A. Cowley. Phys. Rev. 134, 981 (1964)

[30] H.J. Xiang, M. Guennou, J. Íñiguez, J. Kreisel, \& L. Bellaiche. Phys. Rev. B 96, 5, 054102 (2017).

[31] P. Goudochnikov, A.J. Bell. J. Phys.: Condens. Matter. 19, 17, 176201 (2007).

[32] M. Iizumi, J. Axe, G. Shirane, K. Shimaoka. Phys. Rev. B 15, 4392 (1977).

[33] Р. Блинц, Б. Жекш. Сегнетоэлектрики и анти-сегнетоэлектрики: Динамика решетки / Мир, М. (1975). 402 с.

Редактор Ю.Э. Китаев 